\title{
Perfil tiroideo de pacientes eutiroideos con diferentes grados de obesidad
}

\section{Thyroid test results in euthyroid patients with different class of obesity}

\author{
Ximena M. Valdez-Salamea ${ }^{1 *}$, José L. AceVes-Chimal ${ }^{2}$ y Alma Vergara-López ${ }^{1}$
}

'Servicio de Endocrinología; ${ }^{2}$ Servicio de Cirugía Cardiotorácica. Centro Médico Nacional 20 de Noviembre, Instituto de Seguridad y Servicios Sociales de los Trabajadores del Estado (ISSSTE), Ciudad de México, México

\section{RESUMEN}

\begin{abstract}
El hipotiroidismo subclínico es un diagnóstico controvertido en población con sobrepeso y obesidad, se estima que el $25 \%$ de personas con obesidad mórbida tienen niveles elevados de tirotropina (TSH), aunque generalmente por debajo de 10 $\mathrm{mUl} / \mathrm{l}$. Es posible que esto sea el resultado de una respuesta adaptativa de homeostasis tiroidea. Objetivo: Comparar el perfil tiroideo de pacientes eutiroideos con sobrepeso y diferentes grados de obesidad. Material y métodos: Estudio transversal, retrolectivo. Evaluamos 120 pacientes: sobrepeso $(n=30)$, obesidad grado I $(n=30)$, obesidad grado II $(n=30)$ y obesidad mórbida ( $n=30$ ) y comparamos su perfil tiroideo, lipídico y química sanguínea. Resultados: La edad de los pacientes fue de $44 \pm 7$ años; 45 hombres y 75 mujeres. Los niveles de triglicéridos y glucosa fueron significativamente diferentes en el grupo de pacientes con obesidad mórbida $(p<0.05)$. Los niveles de TSH fueron significativamente mayores en el grupo con sobrepeso $(p=0.002)$ y los niveles de triyodotironina total y triyodotironina libre fueron significativamente más elevados en el grupo con obesidad mórbida ( $p=0.01$ y 0.03 respectivamente). Conclusión: En pacientes eutiroideos con diferentes grados de obesidad existen diferencias significativas en el perfil tiroideo, por lo que el diagnóstico de hipotiroidismo subclínico debe ser considerado con cautela.
\end{abstract}

Palabras clave: Sobrepeso. Obesidad. Pruebas de función tiroidea.

\section{ABSTRACT}

Subclinical hypothyroidism is a controversial diagnosis in overweight and obese population; it is estimated that $25 \%$ of morbid obesity patients have elevated thyrotropin (TSH) levels, mostly below $10 \mathrm{mIU} / \mathrm{l}$. It may be result of adaptive thyroid homeostasis. Objective: To compare the thyroid profile of euthyroid patients with that of overweight and with different class of obesity. Material and methods: We conducted a cross-sectional, retrospective study. We evaluated 120 patients: overweight $(n=30)$, obesity class I $(n=30)$, obesity class II $(n=30)$ and morbid obesity $(n=30)$, and compared the thyroid function test results, blood chemistry test and lipid profile. Results: The age of the patients was $44 \pm 7$ years, 45 men and 75 women. Triglyceride and serum glucose levels were significantly different in the group of morbidly obese patients $(p<0.05)$. Serum TSH levels were significantly higher in the overweight group ( $p=0.002$ ) and the total triiodothyronine and free triiodothyronine levels were significantly higher in the morbidly obese group ( $p=0.01$ and 0.03 respectively). Conclusion: In euthyroid patients with varying class of obesity there are significant differences in the thyroid function test results, so the diagnosis of subclinical hypothyroidism should be carefully considered.

Key words: Subclinical hypothyroidism. Obesity. Thyroid profile.

\section{Correspondencia:}

*Ximena M. Valdez-Salamea

E-mail: xime_valdez@msn.com
Fecha de recepción: 27-05-2019

Fecha de aceptación: 07-04-2020

DOI: 10.24875/RME.20001941
Disponible en internet: 03-11-2020 Rev Mex Endocrinol Metab Nutr. 2020;7:167-71

2462-4144 / @ 2020 Sociedad Mexicana de Nutricion y Endocrinologia, AC. Publicado por Permanyer. Éste es un artículo open access bajo la licencia CC BY-NC-ND (http://creativecommons.org/licenses/by-nc-nd/4.0/). 


\section{INTRODUCCIÓN}

De acuerdo con la Encuesta Nacional de Salud y Nutrición de Medio Camino 2016, la prevalencia combinada de sobrepeso y obesidad es del $72.5 \%$ en población adulta, asociándose a varios trastornos metabólicos, entre los que destaca la disfunción del eje hipotálamo-hipófisis-tiroides (HHT). En diversos estudios se ha reportado la creencia equivocada de que el hipotiroidismo se encuentra muy relacionado con la obesidad, de tal manera que actualmente no se recomienda que el índice de masa corporal se utilice como factor determinante para realizar el tamizaje de la función tiroidea ${ }^{1,2,3}$.

Varios estudios han reportado que la leptina tiene un efecto hipofisario directo en la secreción autocrina y paracrina de la liberación de tirotropina $u$ hormona estimulante de la tiroides (TSH), ya que tienen ritmos circadianos casi idénticos, lo que sugiere que la leptina pudiera regular la pulsatilidad y el ritmo circadiano de la TSH. El receptor de leptina se expresa en el núcleo paraventricular, regulando así al eje $\mathrm{HHT}^{4,5,6}$.

El eje HHT actúa como un sistema dinámico adaptativo, que funciona como regulador homeostático para mantener un valor constante de concentraciones séricas de hormonas tiroideas; sin embargo, los niveles de TSH y hormonas tiroideas pueden verse alterados en situaciones fisiológicas y patológicas, en ausencia de cualquier disfunción del sistema de control tirotrópico. En este contexto, algunos autores sugieren que el perfil tiroideo debe ser interpretado con precaución en pacientes con los diferentes grados de obesidad, debido a que pueden existir concentraciones elevadas de TSH como una respuesta adaptativa de homeostasis tiroidea en pacientes con incremento de peso. Esta circunstancia contribuye a la controversia diagnóstica de hipotiroidismo subclínico en esta población ${ }^{2,7,8,9}$.

De Valdés, et al. ${ }^{10}$ observaron en sujetos obesos niveles séricos de TSH tres veces más elevados comparados con personas con peso normal $(5-7 \mathrm{mUl} / \mathrm{ml})$, pero en otros estudios se ha observado que estos niveles usualmente se encuentran por debajo de $10 \mathrm{mUl} / \mathrm{ml}$, argumentando que posiblemente el perfil tiroideo tenga un comportamiento diferente en pacientes con diferentes grados de obesidad, incluyendo pacientes con sobrepeso, especialmente cuando se encuentran metabólicamente eutiroideos $^{11}$. En este estudio comparamos el perfil tiroideo de pacientes eutiroideos con sobrepeso y diferentes grados de obesidad.

\section{MATERIAL Y MÉTODOS}

Realizamos un estudio transversal y retrolectivo, autorizado por los comités de Investigación, Ética y Bioseguridad del Centro Médico Nacional 20 de Noviembre del Instituto de Seguridad y Servicios Sociales de los Trabajadores del Estado. Revisamos los expedientes clínicos de 120 pacientes adultos con sobrepeso $(n=30)$ y diferentes grados de obesidad (grado I, $\mathrm{n}=30$; grado II, $\mathrm{n}=30$ y obesidad mórbida, $\mathrm{n}=30$ ) sin antecedentes de enfermedades tiroideas, ni consumo de medicamentos con efecto en la función tiroidea.

El análisis estadístico se realizó mediante el programa SPSS versión 24.0 para Windows. Para el análisis descriptivo utilizamos media y desviación estándar. Para la comparación entre dos grupos utilizamos prueba ANOVA. La prueba de Tukey se utilizó para identificar el grupo de pacientes que determinó la diferencia en la prueba ANOVA en el contexto de uniformidad de grupos. Se consideró significancia estadística a un valor de $\mathrm{p}<0.05$.

\section{RESULTADOS}

La media de edad de los pacientes fue de $44 \pm 7$ años, 45 del sexo masculino (37\%) y 75 del femenino (63\%). El 51\% tienen diagnóstico de diabetes mellitus (26\% tipo 1 y $25 \%$ tipo 2), con hipertensión arterial sistémica el 30\%, síndrome de ovario poliquístico el $12 \%$ e infertilidad el $7 \%$. El perfil lipídico y la química sanguínea entre los grupos no mostraron diferencias significativas, excepto en los pacientes con obesidad mórbida con niveles 
Tabla 1. Comparación de perfil lipídico y bioquímico

\begin{tabular}{lccccccc}
\hline & Sobrepeso & $\begin{array}{c}\text { Obesidad } \\
\text { grado I }\end{array}$ & $\begin{array}{c}\text { Obesidad } \\
\text { grado II }\end{array}$ & $\begin{array}{c}\text { Obesidad } \\
\text { mórbida }\end{array}$ & $\begin{array}{c}\text { ANOVA } \\
\mathrm{p}\end{array}$ & $\begin{array}{c}\text { Post hoc } \\
\text { grupos }\end{array}$ & $\mathrm{p}^{*}$ \\
\hline $\mathrm{HDL} \mathrm{mg/dl}$ & $43.7 \pm 10.5$ & $45.8 \pm 12.5$ & $42.9 \pm 7.9$ & $46 \pm 10.7$ & 0.60 & - & - \\
\hline $\mathrm{LDL} \mathrm{mg/dl}$ & $122 \pm 40$ & $119 \pm 36$ & $124 \pm 3.9$ & $138 \pm 33$ & 0.22 & - & - \\
\hline Colesterol mg/dl & $202 \pm 69$ & $181 \pm 37$ & $187 \pm 37$ & $191 \pm 39$ & 0.37 & - & - \\
\hline Tg mg/dl & $184 \pm 86$ & $188 \pm 93$ & $180 \pm 93$ & $116 \pm 47$ & 0.002 & $1-3$ vs. 4 & - \\
\hline Creatinina mg/dl & $0.72 \pm 0.07$ & $0.75 \pm 0.03$ & $0.71 \pm 0.01$ & $0.68 \pm 0.02$ & 0.61 & $1-3$ vs. 4 & 0.03 \\
\hline Glucosa mg/dl & $104 \pm 38$ & $116 \pm 54$ & $144 \pm 76$ & $115 \pm 40$ & 0.04 & & - \\
\hline
\end{tabular}

*El análisis post hoc se realizó con la prueba de Tukey.

HDL: lipoproteína de alta densidad; LDL: lipoproteína de baja densidad; Tg: triglicéridos.

Tabla 2. Comparación del perfil tiroideo

\begin{tabular}{|c|c|c|c|c|c|c|c|}
\hline & Sobrepeso & $\begin{array}{l}\text { Obesidad } \\
\text { grado I }\end{array}$ & $\begin{array}{l}\text { Obesidad } \\
\text { grado II }\end{array}$ & $\begin{array}{l}\text { Obesidad } \\
\text { mórbida }\end{array}$ & $\begin{array}{c}\text { ANOVA } \\
\mathrm{p}\end{array}$ & $\begin{array}{l}\text { Post hoc } \\
\text { grupos }\end{array}$ & $\mathrm{p}^{*}$ \\
\hline TSH & $3.86 \pm 1.4$ & $3.4 \pm 1.9$ & $2.93 \pm 1.7$ & $2.3 \pm 1.1$ & 0.002 & $1-3$ vs. 4 & 0.002 \\
\hline T4T & $7.9 \pm 1.3$ & $11.6 \pm 1.5$ & $8.3 \pm 1.5$ & $11.8 \pm 1.9$ & 0.43 & - & - \\
\hline T4L & $0.7 \pm 0.04$ & $0.77 \pm 0.04$ & $0.73 \pm 0.04$ & $0.77 \pm 0.04$ & 0.94 & - & - \\
\hline T3T & $15.5 \pm 3.3$ & $17.3 \pm 2$ & $24.1 \pm 2.7$ & $43 \pm 3.9$ & 0.01 & $1-3$ vs. 4 & 0.009 \\
\hline $\mathrm{T} 3 \mathrm{~L}$ & $0.20 \pm 0.01$ & $0.31 \pm 0.09$ & $0.32 \pm 0.07$ & $0.27 \pm 0.08$ & 0.03 & 1.4 vs. 2.3 & 0.03 \\
\hline$\overline{\mathrm{T} 3 \mathrm{~L} / \mathrm{T} 4 \mathrm{~L}}$ & $0.28 \pm 0.9$ & $0.31 \pm 0.11$ & $0.30 \pm 0.10$ & $0.31 \pm 0.08$ & 0.42 & - & - \\
\hline
\end{tabular}

*El análisis post hoc se realizó con la prueba de Tukey.

TSH: tirotropina u hormona estimulante de la tiroides; T3: triyodotironina; T3T: triyodotironina total; T3L: triyodotironina libre; T4: tiroxina o tetrayodotironina; T4T: tetrayodotironina total; T4L: tetrayodotironina libre.

séricos de triglicéridos y glucosa significativamente mayores $(p<0.05)$ (Tabla 1$)$.

Los niveles séricos de TSH fueron significativamente mayores en el grupo de pacientes con sobrepeso $(p=0.002)$. Los niveles séricos de triyodotironina total (T3T) fueron significativamente mayores en los pacientes del grupo con obesidad mórbida y los niveles de triyodotironina libre (T3L) en los grupos con obesidad grado I y II (Tabla 2).

\section{DISCUSIÓN}

Los trastornos metabólicos y fisiológicos desencadenados por el incremento del peso son procesos de respuesta adaptativa de homeostasis tiroidea, mecanismo conocido como alostasis tipo $2^{8}$; dichos procesos pueden favorecer una confusión en el diagnóstico, sobre todo en el caso del hipotiroidismo subclínico, en el cual la elevación de la TSH por arriba de valores de referencia y niveles de hormonas tiroideas normales prácticamente determinan el diagnóstico.

Se conoce bien que la leptina es producida por los adipocitos y que en condiciones de sobrepeso y grados iniciales de obesidad la producción de leptina se incrementa, esta estimula la transcripción del gen de la hormona liberadora de tirotropina (TRH) y, en consecuencia, la secreción de TRH y TSH, con un incremento en los niveles de hormonas tiroideas circulantes ${ }^{11,12}$, lo cual explica los hallazgos de nuestro estudio, en el que los pacientes con sobrepeso y con grados bajos de obesidad mostraron niveles séricos más elevados de TSH y hormonas tiroideas ${ }^{13}$.

De acuerdo con la estimulación de la leptina en el hipotálamo, sería lógico esperar que en pacientes 
con obesidad mórbida los niveles de TSH se encontraran aún más elevados, sin embargo, la estimulación crónica y elevada de leptina produce en las neuronas del núcleo paraventricular del hipotálamo resistencia a la acción de la hormona, mediante un funcionamiento inadecuado de la cascada de señalización intracelular asociada a la continua activación del receptor, que al mismo tiempo que produce resistencia a su acción anorexigénica, propicia niveles inferiores de TSH comparados con los pacientes con obesidad mórbida ${ }^{12-14}$.

Una vía secundaria de estimulación de la leptina consiste en el incremento de la síntesis del D1 (desyodasa tipo 1) que aumenta la conversión de T4 a T3 con aumento en la relación T3/T4 en tejidos periféricos como el hígado; esto aunado a la disminución de la activación del hipotálamo por la D2 (desyodasa tipo 2) que disminuye la retroalimentación negativa sobre TRH, explica el estado eutiroideo en pacientes analizados en este estudio con sobrepeso y obesidad ${ }^{15}$. A nivel periférico la producción de T3 inversa (rT3) se reduce y la producción de T3 aumenta, incrementando de esta manera el gasto energético y la tasa metabólica basal ${ }^{16-19}$, condición que caracteriza a los pacientes con obesidad, como en nuestro estudio en el que se observó una elevación significativa de las concentraciones de T3T y T3L en pacientes con obesidad mórbida ${ }^{20-22}$.

En pacientes con obesidad grado I y hasta obesidad mórbida se ha descrito hiperactividad del eje tiroideo, haciendo evidente que las hormonas tiroideas son un potente estimulador de la termogénesis adaptativa y que forman parte de los mecanismos autorreguladores de la masa corporal y del almacenamiento de grasa ${ }^{23-26}$; esto explica el aparente estado de normolipidemia y glucemia en nuestros pacientes con obesidad mórbida, que contrastaron significativamente con los pacientes con sobrepeso y obesidad grado I y II, en quienes posiblemente los mecanismos reguladores de energía se encuentran superados por las actividades anhedónicas asociadas al incremento de la masa corporal, descritas en diversas publicaciones psiquiátricas y de cirugía bariátrica ${ }^{27-29}$.

Los niveles bajos de TSH observados en los pacientes con obesidad mórbida comparados con los niveles observados con menores grados de obesidad de este estudio podrían ser explicados por los eventos fisiológicos observados en otros estudios, aunque esto deberá ser demostrado en población mexicana, puesto que existe evidencia de que el comportamiento metabólico difiere de otros grupos étnicos.

En suma, nuestros hallazgos desafían el diagnóstico de hipotiroidismo subclínico en la obesidad, ya que los niveles moderadamente elevados de TSH pueden ser una condición de la actividad metabólica del tejido adiposo, que podría estar presente de forma incipiente en pacientes con sobrepeso, pero que finalmente generan confusión en el momento de establecer el diagnóstico de hipotiroidismo subclínico, lo cual indica que este diagnóstico debe realizarse con precaución en este tipo de pacientes y por lo tanto invita a realizar futuras investigaciones que aclaren con mayor precisión el comportamiento del eje HHT y del estado tiroideo en pacientes con sobrepeso y obesidad, evitando así errores de clasificación y sobretratamiento

\section{LIMITACIONES DEL ESTUDIO}

El estudio consideró un tamaño de muestra piloto estadístico, por lo que la población estudiada es limitada, sin embargo, los hallazgos significativos del estudio sugieren la necesidad de considerar con precaución los parámetros clásicos para establecer el diagnóstico de hipotiroidismo subclínico en pacientes con algún grado de obesidad e invitan a realizar investigaciones con mayor población para confirmar nuestros hallazgos y desarrollar estrategias de mayor precisión diagnóstica de esta entidad patológica.

\section{CONCLUSIONES}

En pacientes eutiroideos con diferentes grados de obesidad existen diferencias significativas en el perfil tiroideo, por lo que el diagnóstico de hipotiroidismo subclínico debe ser considerado con cautela. 


\section{FINANCIAMIENTO}

La presente investigación no ha recibido ayudas específicas provenientes de agencias del sector público, sector comercial o entidades sin ánimo de lucro.

\section{CONFLICTO DE INTERESES}

Los autores declaran no tener conflicto de intereses.

\section{RESPONSABILIDADES ÉTICAS}

Protección de personas y animales. Los autores declaran que para esta investigación no se han realizado experimentos en seres humanos ni en animales.

Confidencialidad de los datos. Los autores declaran que han seguido los protocolos de su centro de trabajo sobre la publicación de datos de pacientes.

Derecho a la privacidad y consentimiento informado. Los autores han obtenido el consentimiento informado de los pacientes y/o sujetos referidos en el artículo. Este documento obra en poder del autor de correspondencia.

\section{BIBLIOGRAFÍA}

1. González-Muniesa P, Martínez-Gonzalez MA, Hu FB, Després JP, Matsuzawa Y, Loos RJF, et al. Obesity. Nat Rev Dis Primers. 2017;3:17034.

2. Heymsfield S, Wadden T. Mechanisms, pathophysiology and management of obesity. N Engl J Med. 2017;376(3):254-66.

3. Encuesta Nacional de Salud y Nutrición de Medio Camino 2016. Informe final de resultados [Internet]. México: Secretaría de Salud; 31 de octubre de 2016. Disponible en: https://www.gob.mx/cms/uploads/attachment/ file/209093/ENSANUT.pdf

4. Mullur R, Liu Y, Brent GA. Thyroid hormone regulation of metabolism. Physiol Rev. 2014;94(2):355-82.
5. Nannipieri M, Cecchetti F, Anselmo M, Camastra S, Niccolini P, Lamacchia $M$, Rossi M, lervasi G, Ferranini E. Expression of thyrotropin and thyroid hormone receptors in adipose tissue of patients with morbid obesity and/or type 2 diabetes: effects of weight loss. Int J Obes (Lond). 2009;33(9):1001-6.

6. Sari R, Balci MK, Altunbas H, Karayalcin U. The effect of body weight and weight loss on thyroid volume and function in obese women. Clin Endocrinol (Oxf). 2003;59(2):258-62.

7. Garvey W, Mechanick J, Brett E, Garber A. Hurley D, Jastreboff A, et al. AACE/ACE Obesity CPG. Endocr Pract. 2016;22(3):10-34.

8. Chatzitomaris A, Hoermann R, Midgley JE, Hering S, Urban A, Dietrich B, et al. Thyroid allostasis-adaptive responses of thyrotropic feedback control to conditions of strain, stress, and developmental programming. Front Endocrinol (Lausanne). 2017;8:163.

9. Nyrnes A, Jorde R, Sundsfjord J. Serum TSH is positively associated with BMI. Int J Obes (Lond). 2006;30(1):100-5.

10. Valdés $S$, Maldonado-Araque $C$, Lago-Sampedro A, Lillo-Muñoz JA García-Fuentes E, Pérez-Valero V, et al. Reference values for TSH maybe inadequate to define hypothyroidism in persons with morbid obesity: Di@bet.es Study. Obesity. 2017;25(4):788-93.

11. Wauters M, Considine RV, Van Gaal LF. Human leptin: from an adipocyte hormone to an endocrine mediator. Eur J Endocrinol. 2000;143(3): 293-311.

12. Sánchez JL. Perfíl fisiológico de la Leptina. Colombia Médica. 2005;36(1):50-9.

13. de Moura Souza A, Sichieri R. Association between serum TSH concentration within the normal range and adiposity. Eur J Endocrinol. 2011;165(1):11-5

14. Reinerh T. Obesity and thyroid function. Mol Cell Endocrinol. 2010;316(2):165-71

15. Friedman JM, Halaas $\mathrm{JL}$. Leptin and the regulation of body weight in mammals. Nature. 1998:395:763-70.

16. Harvey J, Ashford ML. Leptin in the CNS: much more than a satiety signal. Neuropharmacol. 2003;44:845-54

17. Rotondi M, Leporati P, Rizza MI, Clerici A, Groppelli G, Pallavicini C, et al. Raised serum TSH in morbid-obese and non-obese patients: effect on the circulating lipid profile. Endocrine. 2014;45(1):92-7.

18. Srivastava G, Apovian CM. Current pharmacotherapy for obesity. Nat Rev Endocrinol. 2018;14(1)12-24.

19. Peeters RP. Subclinical hypothyroidism. N Engl J Med. 2017;376(26):2556-65.

20. Ortiga-Carvalho TM, Oliveira KJ, Soares BA, Pazos-Moura CC. The role of leptin in the regulation of TSH secretion in the fed state: in vivo and in vitro studies. J Endocrinol. 2002;174(1):121-5.

21. Santini F, Marzullo P, Rotondi M, Ceccarini G, Pagano L, Ippolito S, et al. The crosstalk between thyroid gland and adipose tissue: signal integration in health and disease. Euro J Endocrinol. 2014;171(4):R137-R152.

22. Rosenbaum M, Hirsch J, Murphy E, Leibel RL. Effects of changes in body weight on carbohydrate metabolism, catecholamine excretion, and thyroid function. Am J Clin Nutr. 2000;71(6):1421-32

23. Popovic V, Duntas LH. Leptin TRH and ghrelin: influence on energy homeostasis at rest and during exercise. Horm Metab Res. 2005;37(9):533-7.

24. Ren $R$, Jiang $X$, Zhang $X$, Guan $\mathrm{Q}$, Yu C, Li Y, et al. Association between thyroid hormones and body fat in euthyroid subjects. Clin Endocrinol (Oxf). 2014;80(4):585-90.

25. Macek JZ, Pavelka S, Flachs P, Hensler M, Kus V, Kopecky J. Modulation of type I iodothyronine $5^{\prime}$-deiodinase activity in white adipose tissue by nutrition: possible involvement of leptin. Physiol Res. 2010;59(4):561-9.

26. Grillo CA, Mulder P, Macht VA, Kaigler KF, Wilson FP, Wilson MA, et al. Dietary restriction reverses obesity-induced anhedonia. Physiol Behav. 2014;128:126-32.

27. Van Hout GC, Fortuin FA, Pelle AJ, van Heck GL. Psychosocial functioning, personality, and body image following vertical banded gastroplasty. Obes Surg. 2008:18:115-20.

28. De Panfilis C, Torre M, Cero S, Salvatore P, Dall'Aglio E, Marchesi C, et al. Personality and attrition from behavioral weight-loss treatment for obesity. Gen Hosp Psychiatry. 2008;30:515-2.

29. Guillemot-Legris O, Muccioli GG. Obesity-induced neuroinflammation: Beyond the hypothalamus. Trends Neuroci. 2017;40(4):237-53. 